\title{
NUMERICAL SIMULATION OF WEAKLY COMPRESSIBLE MULTIPHASE FLOWS WITH A BAER-NUNZIATO TYPE MODEL
}

\author{
${\text { Barbara } \mathbf{R e}^{1} \text { and Rémi Abgrall }}^{1}$ \\ ${ }^{1}$ University of Zürich \\ Institute of Mathematics \\ barbara.re@math.uzh.ch
}

Key words: Diffuse interface methods, pressure-based formulation, relaxation parameters, two-phase $\mathrm{CO}_{2}$ flows

\begin{abstract}
We present the results of the simulation of two-phase $\mathrm{CO}_{2}$ flows at low-Mach number, obtained through a pressure-based Baer-Nunziato type model. The underlying full non-equilibrium model enables the description of each phase with its own thermodynamic model, so it circumvents the requirement of the definition of the speed of sound of the vapor-liquid mixture. The primitive formulation, combined with a special pressure scaling to correctly capture the behavior in the zero-Mach limit, is well-suited to model weakly compressible flows, and makes easier the use of arbitrary thermodynamic models. At the interfaces, the phasic velocity and pressure are driven toward the equilibrium by means of relaxation processes, whose velocities are controlled by user-defined parameters. The set of seven partial differential equations describing the flow evolution is discretized through a finite-volume scheme in space and an hybrid implicit-explicit time discretization, to avoid the stringent time step limitation imposed by the acoustics. We compare the results of a shock-tube problem, initially containing saturated $\mathrm{CO}_{2}$, obtained according to the stiffened gas model and to the Peng-Robinson equation of state.
\end{abstract}

\section{INTRODUCTION}

Among the technologies able to contrast the global warning, carbon capture and storage (CCS) is regarded as a crucial and effective approach. Consequently, the numerical investigation of carbon dioxide $\left(\mathrm{CO}_{2}\right)$ flows under the different conditions we can encounter within the CCS framework is becoming more and more important. In this work, we focus in particular in unsteady weakly compressible twophase flows. Such kind of flows may occur in the transport pipelines, because of fluctuating in the $\mathrm{CO}_{2}$ supply, impurities, or during transient events, such as start-up, shut-down or depressurization [1]. From a numerical point of view, these flows present different challenging aspects. First of all, the weak compressibility - that is the condition where the flow velocity is considerably smaller than the speed of sound but compressibility effects cannot be neglected-makes inefficient and inaccurate the standard compressible solvers. Second, the multitude of spatial scales and the presence of dynamic interfaces that separate the different phases call for an effective modeling that avoids the full resolution of the flow field but takes into consideration the relevant flow features. Third, a flexible implementation of the thermodynamic modeling for the $\mathrm{CO}_{2}$ is recommended to be able to customize it according to the different applications. 
To answer these demands, we propose here a pressure-based multiphase solver based on a non-equilibrium Baer and Nunziato (BN)-type model. In this model, no equilibrium is assumed between phase pressure, velocity, nor internal energy and the flow dynamics is described by an hyperbolic relaxation system, where closure terms model the phase interaction and source terms control how phase equilibrium is reached. Although the large number of equations and the consequent complexity could hinder the use of BN-type models, they present some peculiar features we consider beneficial for this work. First of all, each phase is described by its own thermodynamic model and it is not required to define a speed of sound for the mixture, preventing possible issues related to its continuity at phase boundaries. Then, according to a diffuse-interface method (DIM) approach, the interfaces are not explicitly tracked, but they are modeled as thin, numerically diffuse zones, and this is important to easily deal with moving interfaces in unsteady simulations. Moreover, since each numerical cell contains at least an arbitrarily small amount of all phases, the same numerical method can be used in every cell, regardless whether it contains this or that phase or both [2]. Finally, the model contains several parameters, e.g., the relaxation terms, which could be tuned according to experimental data or to represent different flow topologies.

The numerical tool we develop here is based on a previous work [3], in which we derive a pressure-based BN-type model, particularly suited to flows at low Mach number. In this work, we discuss the relaxation process, both considering finite and infinite relaxation parameters, and we compare the results obtained by different thermodynamic models for $\mathrm{CO}_{2}$.

The paper structure is the following. In the first part of Sec. 2, we briefly recall the pressure-based BNtype model for weakly compressible flows and the adopted thermodynamic models. Then, we discuss the

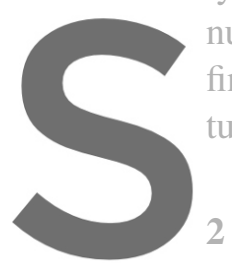
numerical discretization and, in Sec. 2.2.2, we exp
finite and infinite parameters. Finally, Sec. 3 presen
tube test involving $\mathrm{CO}_{2}$ at saturated conditions and
2 METHODOLOGY
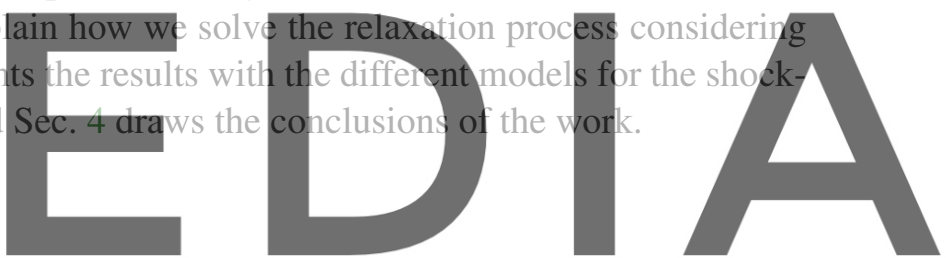

The model we use to describe the two-phase flow is derived from the symmetric variant of the Baer and Register fQr frea at

for the balance of mass, momentum and energy for each fluid, and an evolution equation for the volume

fraction $\alpha$ of one of the phases. The volume fraction of other phase is simply computed from $\alpha_{1}+\alpha_{2}=1$, where the subscripts 1 or 2 refer to the phase, but, being the model symmetric, there is no distinction between liquid or vapor phase.

In this work, instead of solving the equations for the total energy (a conservative variable), we solve the equations describing the evolution of the pressure (a primitive variable), of each phase. This choice helps to prevent the occurrence of spurious pressure oscillations and makes easier the use of arbitrary equations of state $[4,5]$. The two-phase, pressure-based, BN-type model reads 


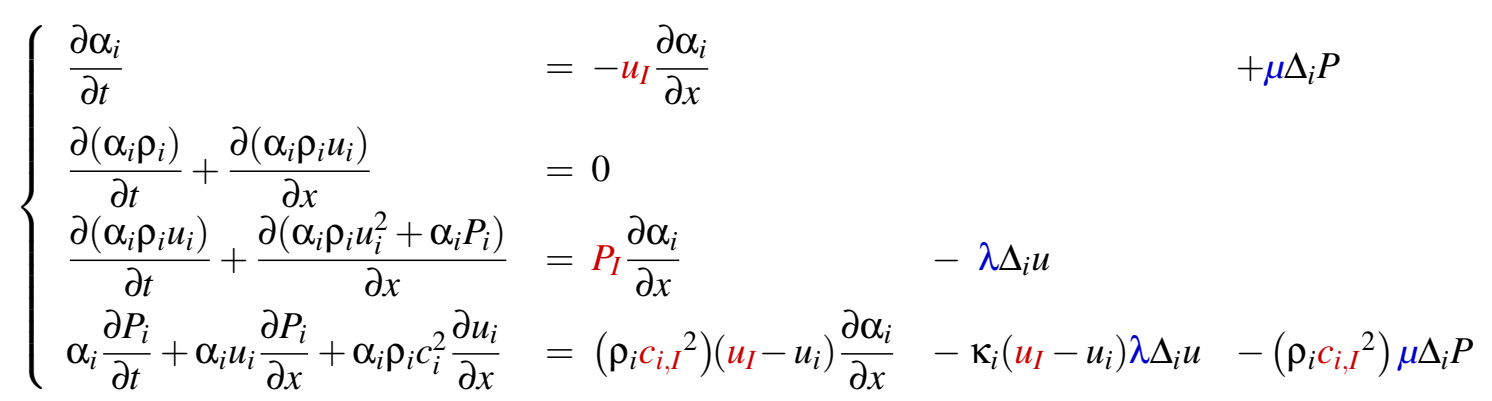

where the last three equations are repeated also for the other phase. The variables are $\alpha$ the volume fraction, $\rho$ the density, $u$ the velocity and $P$ the pressure, and the subscript $i$ refers to the phase (if indicated after a group of variables, it applies to whole group, e.g., $\left.\alpha \rho_{i}=\alpha_{i} \rho_{i}\right)$. On the left-hand side of the equations, we have the terms similar to the ones in the Euler equations, while on the right-hand side we have the non-conservative and the source terms. In red, we highlight the closure terms for the interface velocity $u_{\mathrm{I}}$ and interface pressure $P_{\mathrm{I}}$, which are defined as the weighted averages of the phasic velocity and pressure as in $[2,6]$, and the term $c_{i, I}$ which represents a kind of interface speed of sound, as defined in the following paragraph. As concerns the velocity and the pressure relaxation, the operators $\Delta u$ and $\Delta P$ denote the jumps in each variable between the phases $\left(\Delta_{1} u=u_{1}-u_{2}, \Delta_{2} P=P_{2}-P_{1}\right.$, etc.), while the parameters $\lambda$ and $\mu$, in blue, determine the velocity of relaxation processes. In this work, we consider both the case of finite and of infinite relaxation parameters.
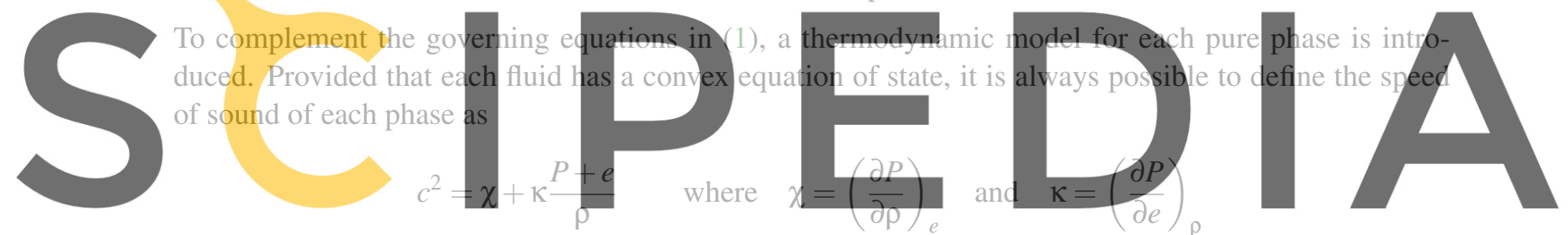

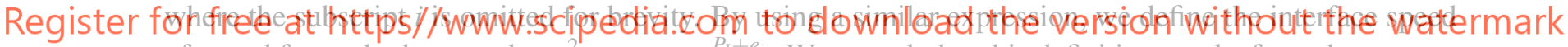
of sound for each phase so that $c_{i, I}^{2}=\chi_{i}+\kappa_{i} \frac{P_{I}+e_{i}}{\rho_{i}}$. We remark that this definition results from the process of deriving the pressure equations of the BN-type model [3], but it has no thermodynamic meaning and, given a pressure interface, can be always defined, independently from the adopted thermodynamic model.

In this work, we consider two thermodynamic models: the stiffened gas and the Peng-Robinson equation of state. Widely used for vapor-liquid flows, the stiffened gas model can be considered an extension of the polytropic ideal gas able to take into consideration the repulsive and agitation molecular effects [7]. The pressure and the internal energy (for a single phase) are given by

$$
P(e, \rho)=(\gamma-1) e-\gamma P_{\infty}-(\gamma-1) \rho q, \quad e(T, \rho)=c_{\nu} \rho T+\rho q+P_{\infty}
$$

where the ratio of specific heats $\gamma$, the specific heat capacity at constant volume $c_{v}$, the parameters $P_{\infty}$ and $q$ are constants that depend on the material and can be determined by fitting experimental data, e.g., the saturation curve [8]. Despite its simplicity, the stiffened gas model is relatively accurate in the region close to the chosen reference point. The values for the $\mathrm{CO}_{2}$ used in this work are taken from [9] and summarized in Table 1. 
Table 1: Stiffened gas parameters for the $\mathrm{CO}_{2}$ used in this work, as given [9].

\begin{tabular}{lcccc}
\hline & $\gamma[-]$ & $P_{\infty}[\mathrm{Pa}]$ & $c_{v}[\mathrm{~J} / \mathrm{kg} \mathrm{K}]$ & $q[\mathrm{~J} / \mathrm{kg}]$ \\
\hline Liquid & 1.23 & $1.3210^{8}$ & 2440 & $-6.2310^{5}$ \\
Vapor & 1.06 & $8.8610^{5}$ & 2410 & $-3.0110^{5}$ \\
\hline
\end{tabular}

Cubic equations of state (EOS), such as Peng-Robinson (PG) [10], are widely used in industrial applications as they combined computational efficiency, robustness and accuracy. In this work, we use the particular implementation of the Peng and Robinson model, provided by an in-house thermodynamic library developed at SINTEF Energy [11], specifically suited for vapor-liquid $\mathrm{CO}_{2}$ flows. This version, with respect to the standard Peng and Robinson EOS, exploits the corresponding state concept to enhance the accuracy of certain properties, such as the density and the speed of sound for the liquid phase.

\subsection{Low-Mach scaling}

Being interested in the simulation of weakly-compressible flows, we introduce a special scaling of the equations (1), to recover the correct scaling of the pressure with the Mach number $M$, for $M \rightarrow 0$ [12]. The idea is to define the dimensionless pressure as $P=\frac{\tilde{P}-P_{\mathrm{r}}}{\rho_{\mathrm{r}} u_{\mathrm{r}}^{2}}$, where $\tilde{P}$ is the dimensional pressure, $P_{\mathrm{r}}$ is a reference (dimensional) pressure, while $\rho_{\mathrm{r}}$ and $u_{\mathrm{r}}$ are the reference (dimensional) density and velocity, as in standard adimensionalization. According to this modeling choice, we are filtering out the acoustics, while the dimensionless pressure $P$ is responsible to fulfill the pressure equations, but it

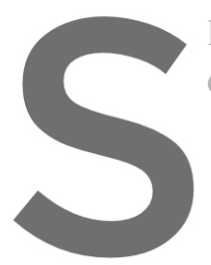
looses its thermodynamic equation, which, for phase
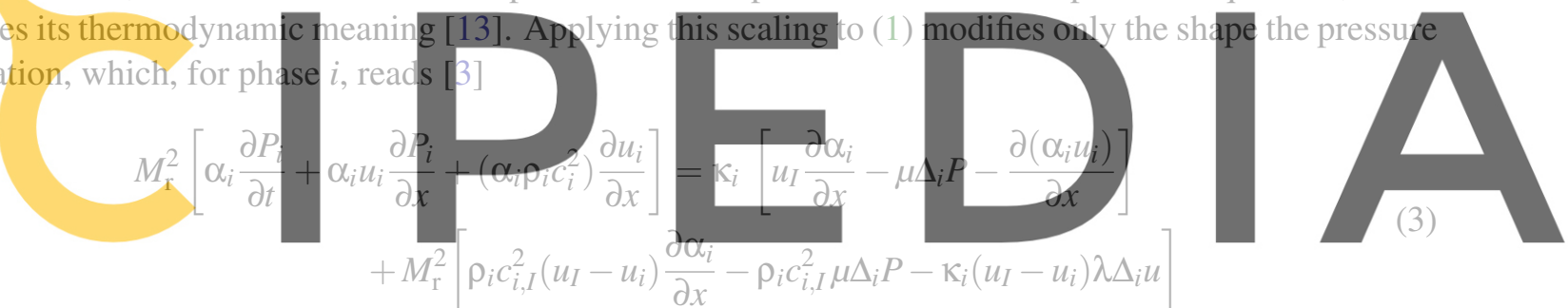

Register for free at https//www.scipedia.com to download the version without the watermark where $M_{\mathrm{r}}$ is the reference Mach number, defined as $M_{\mathrm{r}}^{2}=\frac{\rho_{\mathrm{r}} u_{\mathrm{r}}^{2}}{P_{\mathrm{r}}}$ amd representative of the global level of compressibility of the problem under investigation. This equation has the nice feature that, for $M_{\mathrm{r}} \rightarrow 0$, only the terms in the first square brackets of the right-hand side play a role, which, taking into consideration the equation of the volume fraction evolution, impose that $\frac{\partial \alpha_{i}}{\partial t}+\frac{\partial\left(\alpha_{i} u_{i}\right)}{\partial x}=0$. This is the desired incompressibility constraint on the velocity, which guarantees the recover of the correct pressure behavior in the zero-Mach limit.

\subsection{Numerical discretization}

The complete model consists in the volume fraction equation in (1), the mass and momentum equations in (1) for each phase, and the the pressure equation (3) for both phases. We solve this set of seven equations by using the Strang splitting approach: first, we compute the solution of the hyperbolic part, that is left-hand side of the equations plus the non-conservative terms, then we take into consideration the source terms. 


\subsubsection{Hyperbolic operator}

The hyperbolic operator is based on an implicit-explicit time integration, which integrates implicitly the acoustic terms but explicitly the convective ones. This choice represents a compromise between efficiency and complexity: the time step limitation imposed by the CFL condition is mitigated, as it depends only on the convective velocities $u$, but it is independent from the speeds of sound (and in low Mach problems $u_{i} \ll c_{i}$ ), and the equations exhibit only a mild non-linearity, which is treated by a Newton's linearization. As concerns the spatial discretization, we use a finite-volume approach over a staggered grid. The hyperbolic operator is described in [3], so we report here only the main features.

As done commonly in pressure-based, uniform-Mach schemes [14, 15], we solve the equations in a segregated way. In particular, we split the momentum equations in two steps: first, we compute an intermediate value $\alpha \rho u_{i}^{*}$ by considering explicitly the pressures (i.e., by using $P_{i}^{n}$ instead of $P_{i}^{n+1}$ ), then we correct the momentum from $\alpha \rho u_{i}^{*}$ to $\alpha \rho u_{i}^{n+1}$ by taking into account the contributions due to the pressure correction $\delta P_{i}=P_{i}^{n+1}-P_{i}^{n}$ [3]. Hence, at the time step $t^{n+1}$

\section{1. we solve the equation for the volume fraction $\alpha_{1}$, considering the velocities at $t^{n}$,}

\section{2. we solve the equations for $\alpha \rho_{1}$ and $\alpha \rho_{2}$,}

3. we compute, per each phase, the intermediate value of the momentum $\alpha \rho u_{i}^{*}$,

4. we solve both pressure equations in a coupled way, by considering implicitly the velocity divergence, that is by introducing here an approximation of the velocities $u_{i}^{n+1}$ obtained from the momentum correct

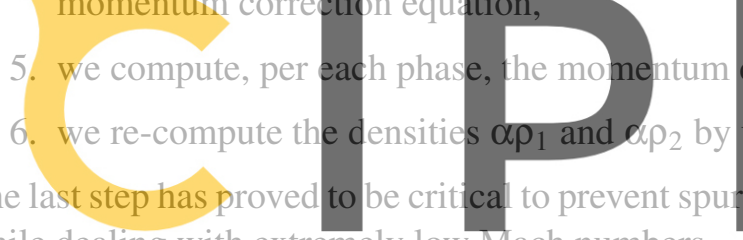

while dealing with extremely low Mach numbers.

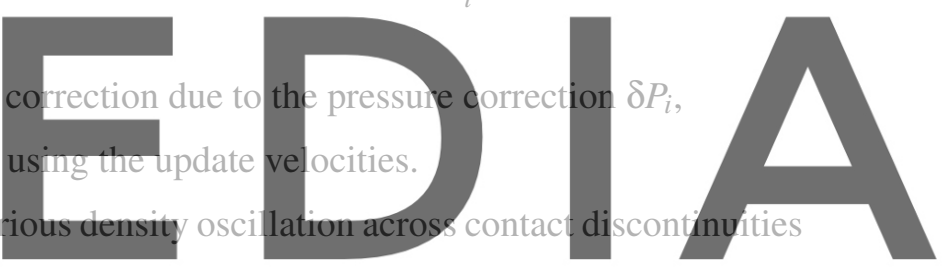

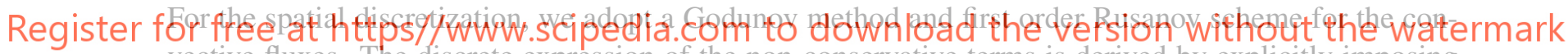
vective fluxes. The discrete expression of the non-conservative terms is derived by explicitly imposing the fulfillment of the non-disturbance condition, according to which a two-phase flow initially uniform in pressure and velocity should stay uniform in these variables also while evolving in time [16]. The resulting expressions of the non-conservative terms are given in [3].

\subsubsection{Source operator}

We account for the source terms responsible for velocity and pressure relaxation by solving two ODE problems: the first one evolves the solution obtained by the hyperbolic operator by imposing the velocity relaxation, the second one imposes the pressure relaxation, starting from the solution of the first ODE problem. Gathering the relaxation terms in two vectors, $\mathbf{R}_{\lambda}$ and $\mathbf{R}_{\mu}$, the discrete ODE problems can be written as

$$
\frac{\mathrm{U}^{\triangleright}-\mathrm{U}^{\triangleright}}{\Delta t}=\mathbf{R}_{\lambda} \Delta u, \quad \frac{\mathrm{U}^{\star}-\mathrm{U}^{\diamond}}{\Delta t}=\mathbf{R}_{\mu} \Delta P
$$


where $\mathrm{U}$ is the vector of solution variables, and the superscripts $\triangleright, \diamond$, and $\star$ denotes the solution resulting from, respectively, the hyperbolic operator, the velocity relaxation, and the pressure relaxation.

As mentioned before, we consider here both finite and infinite relaxation. In the former case, the ODE system for the velocity relaxation yields

$$
\Delta_{1} u=\left(u_{1}^{\triangleright}-u_{2}^{\triangleright}\right) \frac{\alpha \rho_{1} \alpha \rho_{2}}{\alpha \rho_{1} \alpha \rho_{2}+\lambda \Delta t\left(\alpha \rho_{1}+\alpha \rho_{2}\right)} \quad \text { with } \quad \alpha \rho_{i}=\alpha \rho_{i}^{\diamond}=\alpha \rho_{i}^{\triangleright} \quad \text { and } \quad \alpha_{i}^{\diamond}=\alpha_{i}^{\triangleright},
$$

from which the variation on the other variables can be straightforwardly computed. The solution of the ODE problem modeling the pressure relaxation is not so trivial, because of the highly non-linearity resulting from the thermodynamics. For this reason, we introduce an approximation and evaluate all thermodynamic variables at the known state $U^{\diamond}$. The resulting non-linear system, composed by three equations for $\alpha_{1}, P_{1}$, and $P_{2}$, is solved by using the non-linear solvers available in the scientific library PETSc [17].

For the instantaneous relaxation, that is when we consider $\lambda$ and $\mu$ as infinite, we proceed following [2]. The velocity relaxation, which does not modify the densities and the volume fraction, gives the equilibrium velocity $u^{\diamond}=u_{1}^{\diamond}=u_{2}^{\diamond}=\frac{\alpha \rho u_{1}^{\diamond}+\alpha \rho u_{2}^{\diamond}}{\alpha \rho_{1}+\alpha \rho_{2}}$. Then, to update consistently the pressure of each phase, we approximate as in [2] the interface velocity as $\overline{u_{I}}=\frac{1}{2}\left(u_{I}^{\triangleright}+u_{i}^{\diamond}\right)$ and we consider it constant during the integration. This yields to

$$
P_{i}^{\diamond}=P_{i}^{\triangleright}-\rho_{i} \frac{\kappa_{i}}{2}\left(u_{I}^{\triangleright}-u_{i}^{\triangleright}\right)\left(u^{\diamond}-u_{i}^{\triangleright}\right)
$$

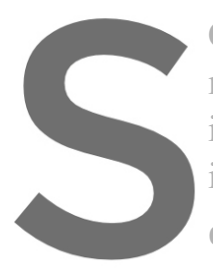

Concerning the pressure mimicking again [2], w iteratively. First of all, integrate them. While con $\alpha_{i}$ is unknown, while $T_{1 i}$
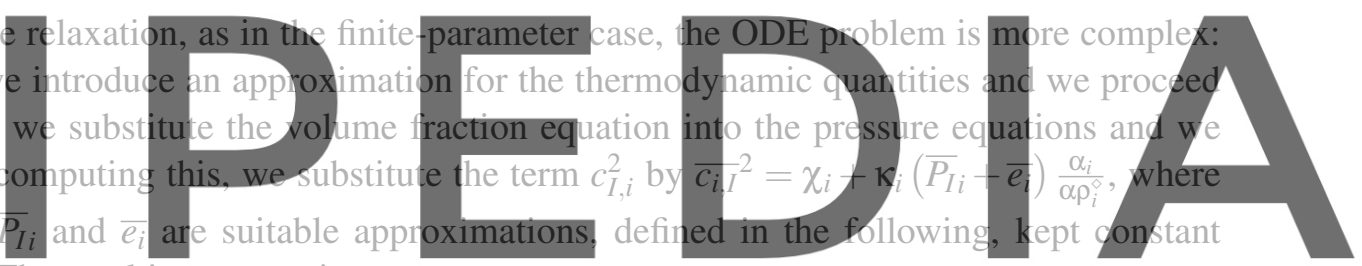

during the integration. The resulting expressions are

Register for free at https//www.scipedia.com to download the version without the watermark

$$
M_{\mathrm{r}}^{2}\left(P_{2}^{\star}-P_{2}^{\diamond}\right)=M_{\mathrm{r}}^{2} \chi_{2}\left(\alpha \rho_{2}\right) \diamond\left(\frac{1}{1-\alpha}-\frac{1}{\alpha_{2}^{\diamond}}\right)-\left[M_{\mathrm{r}}^{2}\left(\kappa_{2} \bar{P}_{I 2}+\bar{e}_{2}\right)+\kappa_{2}\right]\left(\log (1-\alpha)-\log \left(\alpha_{2}^{\diamond}\right)\right.
$$

where the only unknown in the right-hand side is $\alpha=\alpha_{1}^{\star}$. So, we look iteratively for the value of $\alpha$ that solves $f(\alpha)=P_{1}^{\star}(\alpha)-P_{2}^{\star}(\alpha)=0$. At each iteration $k$, we re-compute the pressures $P_{i}^{k}=P_{i}\left(\alpha^{k}\right)$ and the approximation $\overline{P_{I}}=\frac{1}{2}\left(P_{I}^{\triangleright}+P_{i}^{k}\right)$ and $\overline{e_{i}}=\frac{1}{2}\left(e_{i}\left(\rho_{i}^{k}, P_{i}^{k}\right)+e_{i}^{\diamond}\right)$, where we evaluate $e_{i}$ through the equations of state using $\rho_{1}^{k}=\alpha \rho_{1}^{\diamond} / \alpha^{k}$ and $\rho_{2}^{k}=\alpha \rho_{2}^{\diamond} /\left(1-\alpha^{k}\right)$.

\section{RESULTS}

This section show the results of the simulations of a shock-tube problem filled with $\mathrm{CO}_{2}$ at saturation conditions $^{1}$. In the left chamber the saturation temperature is $T_{L}=260 \mathrm{~K}$ (corresponding to a pressure $P_{L}=23.98 \mathrm{bar}$ ), while in the right chamber it is $T_{R}=280 \mathrm{~K}$ (corresponding to a pressure $P_{L}=41.5 \mathrm{bar}$ ).

\footnotetext{
${ }^{1}$ In thermodynamics, a saturation condition is a state lying along the vapor-liquid equilibrium curve
} 
The mixture composition is different: on the left, the liquid volume fraction is $\left(\alpha_{1}\right)_{L}=0.75$, while on the right $\left(\alpha_{1}\right)_{R}=0.25$. Initially, the liquid and the vapor are at rest and they have the same temperature and pressure. When the diaphragm between the chambers is removed, the flow starts to interact. We compute the solution after $0.05 \mathrm{~s}$, using $N_{t}=500$ time steps and discretizing the domain $-60 \mathrm{~m} \leq x \leq 60 \mathrm{~m}$ (the initial discontinuity position is at $x=0$ ) with a uniform grid of 1200 cells.

We compute the results using different sets of relaxation parameters and thermodynamic models. Figure 1 shows the profiles of the volume fraction, the mixture density, the pressures and the velocities computed in different tests:

- blue test: Peng-Robinson equation of state and the mild set of finite relaxation parameters;

- orange test: Peng-Robinson equation of state and the strong sets finite relaxation parameters;

- yellow test: Stiffened gas model and the mild set of finite relaxation parameters;

- violet test: Stiffened gas model and the strong set of finite relaxation parameters;

- green test: Stiffened gas model and infinite relaxation parameters.

The sets of finite relaxation parameters are defined in Table 2. Figure 2 displays some zoomed views of these results. As expected, because of the mild pressure jump, the flow velocities are pretty low, especially compared to the speed of souns. Indeed, the Mach number is everywhere below 0.1.

First of all, we have to say that, starting from the same initial pressure and temperature, different thermodynamic models generate different values for the density, as it can be seen in the top right pane of

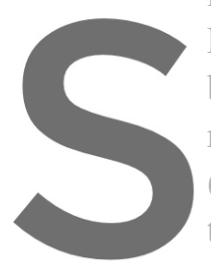
Fig. 1, especially for the left between different models, relaxation parameters are (e.g., see the expansion wa two thermodynamic models give different values o
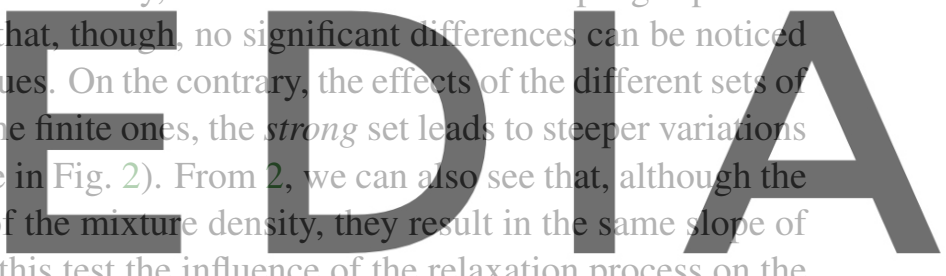

the density variation. This probably means that in this test the influence of the relaxation process on the

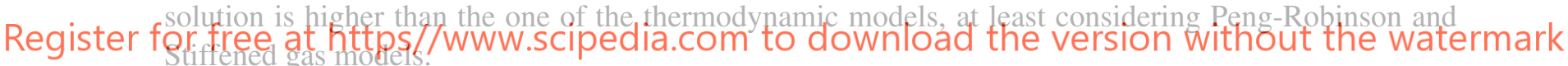

Concerning the magnitude of the relaxation parameters, both the mild and the strong sets drive, as expected, the phasic pressures toward the equilibrium. This is not true for the velocity, because the smallest value of $\lambda$ is somehow not sufficient for a match, as shown by the not-overlapping blue and yellow lines in Fig. 2 (bottom-right picture).

In addition, we have repeated the test also imposing instantaneous relaxation (for brevity, only with the stiffened gas). By comparing these results (in green in Figs. 1 and 2, we can say that this process gives significantly different results from the finite relaxation. Notably, it generates faster waves, so even the positions, not only the magnitude, of the relevant flow features are different.

The results of the orange test are plotted also in Fig. 3, which displays the thermodynamic plane $P-v$ with the saturation curve. This picture gives a clear idea about where the test takes place and how the relaxation process handles the phasic equilibrium. 
Table 2: Sets of finite relaxation parameters used in this work.

\begin{tabular}{lcc}
\hline & $\lambda\left[\mathrm{kg} /\left(\mathrm{m}^{3} \mathrm{~s}\right)\right]$ & $\mu[\mathrm{ms} / \mathrm{kg}]$ \\
\hline Mild & $10^{4}$ & $10^{1}$ \\
Strong & $10^{10}$ & $10^{7}$ \\
\hline
\end{tabular}

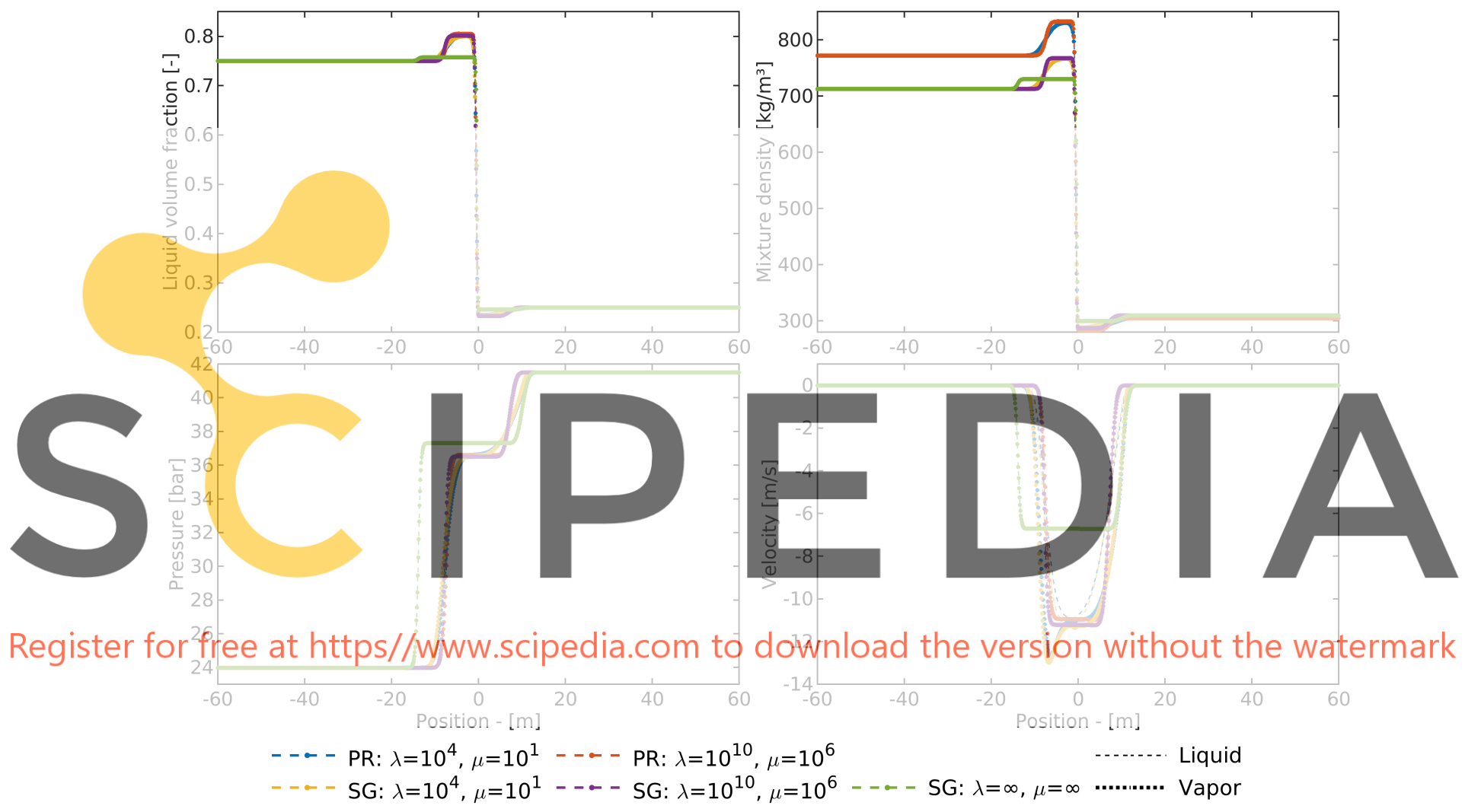

Figure 1: Results of the $\mathrm{CO}_{2}$ shock-tube, at $t_{F}=0.05 \mathrm{~s}$ in five different simulations. The blue and orange results are computed assuming the Peng-Robinson (PR) equation of state, using two different sets of finite relaxation parameters, the mild and the strong one, respectively (the units are not reported for brevity, but are given in Table 2). The yellow and the violet results are computed assuming the Stiffened-Gas (SG) model, using the same two different sets of finite relaxation parameters, that is the mild and the strong one, respectively. The green results are still computed assuming the Stiffened-Gas (SG) model, but imposing instantaneous relaxation, that is $\lambda=\infty$ and $\mu=\infty$. In the top row, we have the liquid volume fraction $\alpha_{1}$ and the mixture density $\alpha \rho=\alpha \rho_{1}+\alpha \rho_{2}$. In the bottom row, we have the pressure and the velocity for each phase: in these plots, the color distinguishes the test, whereas the line format distinguishes the phases, with the liquid phase shown by thin dashed line and the vapor phase by ticker dot markers. 


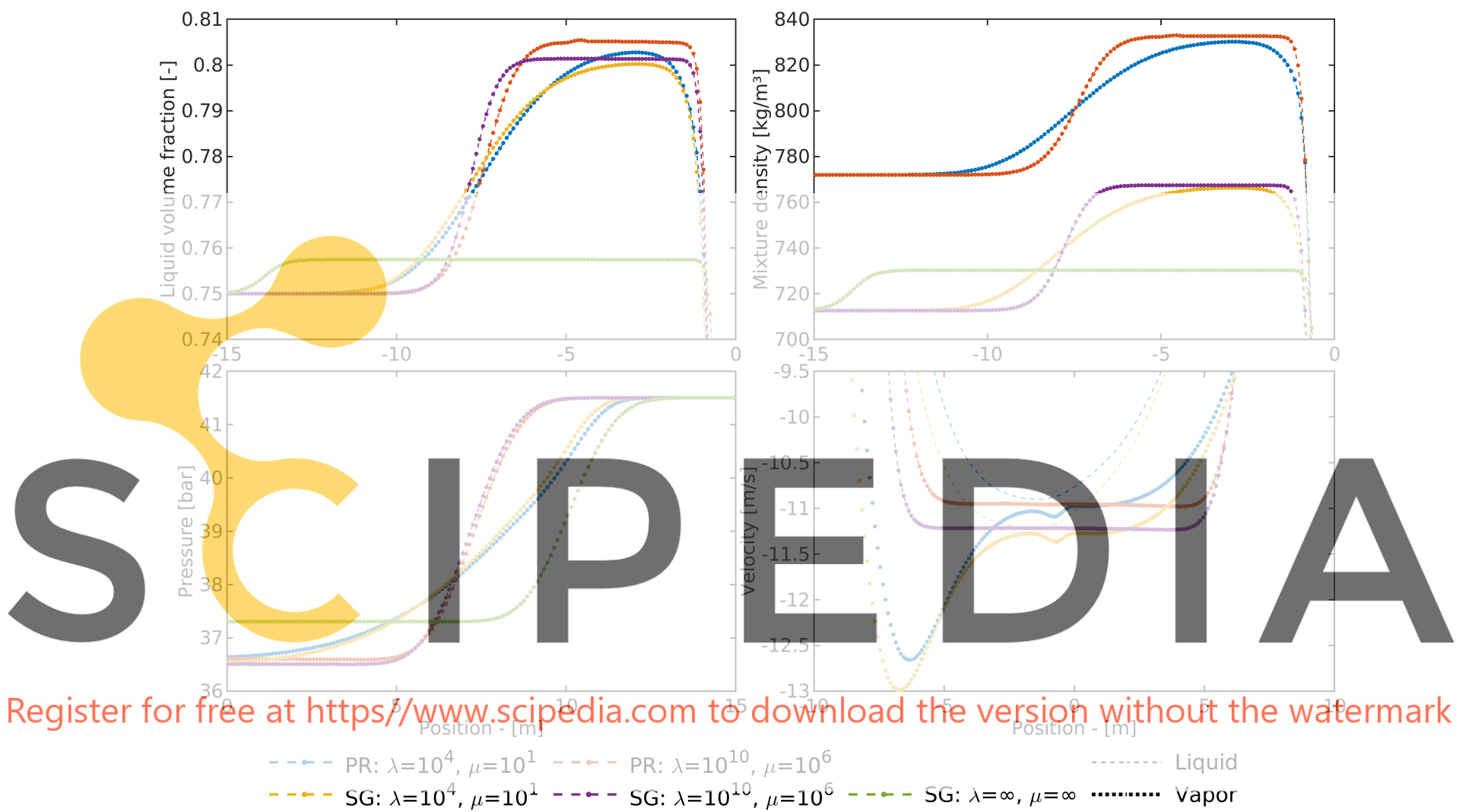

Figure 2: Results of the $\mathrm{CO}_{2}$ shock-tube, at $t_{F}=0.05 \mathrm{~s}$ in five different simulations, as described in Fig. 1. Here, the details for some portion of the grid are shown. In the top row, the liquid volume fraction and the mixture density are shown for $x=[-15,0]$, so in the left chamber. In the bottom row, the pressure in the right chamber close to the expansion fan is shown, along with the velocity in the region across the initial discontinuity. 


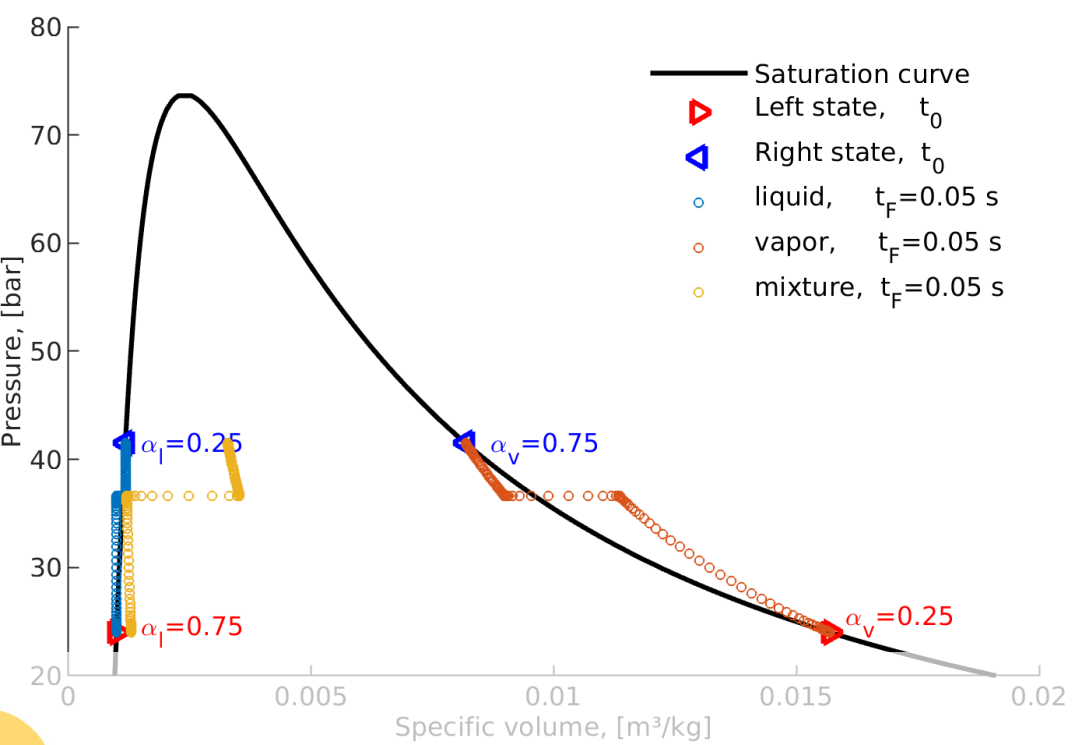

Figure 3: Results of the $\mathrm{CO}_{2}$ shock-tube over the thermodynamic plane $P-v$. The Peng-Robinson equation of state and the strong set of finite relaxation parameters (defined in Tab. 2) are used. Triangular marks refers to the initial conditions. The flow field at $t_{F}=0.05 \mathrm{~s}$ is displayed by circle marks: cyan ones refer to the liquid phase, dark orange to the vapor phase, and dark yellow to the mixture.

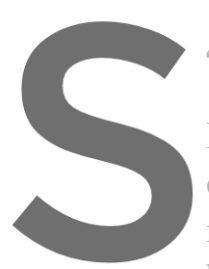

\section{CONCLUSIONS}

In this work, we have presented the

obtained according to a pro

metric Baer and Nunziato one, w
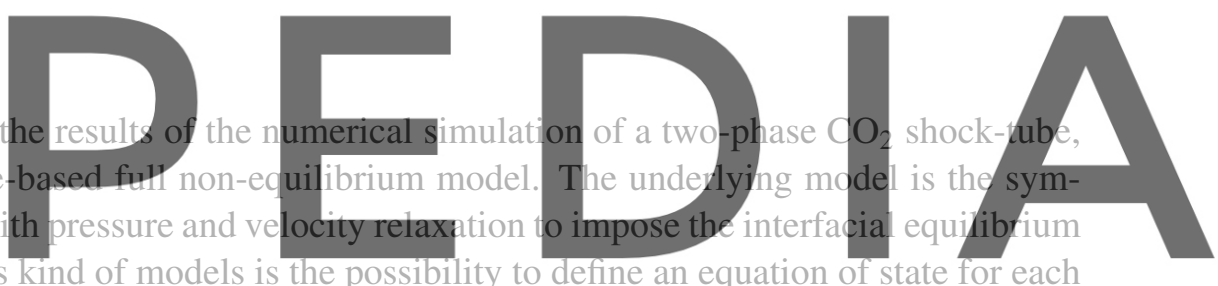

between phases. A benefit of this kind of models is the possibility to define an equation of state for each

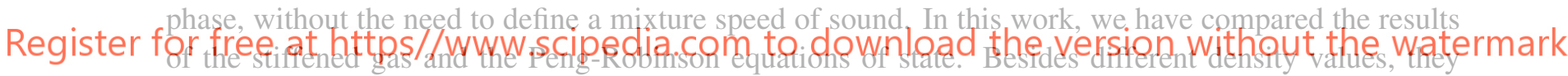

give comparable results for the conditions under investigation. In addition, we have compared different sets of finite relaxation parameters: the main differences can be observed in the velocities, which do not match when using $\lambda=10^{4}$, but they do for $\lambda=10^{10}$. This result does not undermine the validity of the method, but can be seen as an additional degree of freedom it provides. Finally, even if larger values of the relaxation parameters lead to the phasic equilibrium, the results of this process are different from the instantaneous relaxation process. This is probably due to the different approximations, concerning especially the thermodynamics, introduced while solving the ODE systems for the relaxation terms. Currently, we are studying the possibility to avoid some of these simplifications, to reach more accurate results.

\section{Acknowledgments}

This publication has been produced with support from the NCCS Centre, performed under the Norwegian research program Centres for Environment-friendly Energy Research (FME). The authors acknowledge the following partners for their contributions: Aker Solutions, ANSALDO Energia, CoorsTek Membrane 
Sciences, EMGS, Equinor, Gassco, KROHNE, Larvik Shipping, Norcem, Norwegian Oil and Gas, Quad Geometrics, Shell, TOTAL, and the Research Council of Norway (257579/E20).

B. Re gratefully acknowledge also the support of the "Forschungskredit of the University of Zurich, grant no. [FK-20-121]".

\section{REFERENCES}

[1] P. Aursand, M. Hammer, S.T. Munkejord, $\emptyset$. Wilhelmsen. Pipeline transport of $\mathrm{CO}_{2}$ mixtures: Models for transient simulation. Int. J. Greenh. Gas Control (2013). 15: 174-185.

[2] R. Saurel and R. Abgrall. A Multiphase Godunov Method for Compressible Multifluid and Multiphase Flows. J Comput Phys (1999). 150(2):425-467

[3] B. Re and R. Abgrall. Non-equilibrium Model for Weakly Compressible Multi-component Flows: the Hyperbolic Operator. In Non-Ideal Compressible-Fluid Dyn. Propuls. Power (F. Di Mare, A. Spinelli, and M. Pini, eds.), Springer, 2019.

[4] S. Karni. Hybrid multifluid algorithms. SIAM J Sci Comput (1996). 17(5):1019-1039.

[5] Y. Lv. Development of a nonconservative discontinuous Galerkin formulation for simulations of unsteady and turbulent flows. Int J Numer Methods Fluids (2020). 92:325-346.

[6] A. Zein, M. Hantke, and G. Warnecke. Modeling phase transition for compressible two-phase flows applied to metastable liquids. J Comput Phys (2010). 229: 2964-2998

[7] F. Harlow and A. Amsden. Fluid Dynamics, in: Monographe LA, vol. 4700, Los Alamos National Laboratory, Los Alamos, NM, 1971.

[8] O. Le Mtayer and R. Saurel. The Noble-Abel Stiffened-Gas equation of state. Physics of Fluids (2016). 28(4):046102

[9] H. Lund and P. Aursand. Two-Phase Flow of $\mathrm{CO}_{2}$ with Phase Transfer. Energy Procedia (2012). 23: $246-255$

[10] D.Y. Peng and D.B. Robinson. A new two-constant equation of state. Ind Eng Chem Fund (1976) 15(1):59-64.

[11] Ø. Wilhelmsen, A. Aasen, G. Skaugen, P. Aursand, A. Austegard, E. Aursand, ... M. Hammer. Thermodynamic modeling with equations of state: present challenges with established methods. Ind Eng Chem Res (2017). 56(13):3503-3515

[12] I. Wenneker, A. Segal, and P. Wesseling. A Mach-uniform unstructured staggered grid method. Int J Numer Methods Fluids (2002). 40(9):1209-1235

[13] C.D. Munz, S. Rollaer, R. Klein, and K.J. Geratz. The extension of incompressible flow solvers to the weakly compressible regime Computers \& Fluids (2003). 32:173-196

[14] H. Bijl and P. Wesseling. A Unified Method for Computing Incompressible and Compressible Flows in Boundary-Fitted Coordinates. J Comput Phys (1998). 141(2):153-173

[15] N. Kwatra, J. Su, J.T. Grtarsson, and R. Fedkiw. A method for avoiding the acoustic time step restriction in compressible flow. J Comput Phys (2009). 228(11):4146-4161 
[16] R. Abgrall. How to Prevent Pressure Oscillations in Multicomponent Flow Calculations: A Quasi Conservative Approach. J Comput Phys (1996). 125(1):150-160

[17] S. Balay, S. Abhyankar, M. Adams, J. Brown, P. Brune, K. Buschelman, ... H. Zhang. PETSc Web page (2019). http: / /www. mcs . anl.gov/petsc

[18] B. Re and R. Abgrall. Numerical simulation of weakly compressible multiphase flows with a Baer-Nunziato type model. WCCM-ECCOMAS Congress, online, Jan 11-14 2021. https: //slideslive.com/38944423 